\title{
Renoprotective effects of extracellular vesicles: a systematic review
}

\author{
Hossein Abbaszadeh ${ }^{1}$, Farzaneh Ghorbani², Mehdi Talebi², Mehdi Yousefi ${ }^{2}$, and Ali Akbar \\ Movassaghpour ${ }^{2}$ \\ ${ }^{1}$ Affiliation not available \\ ${ }^{2}$ Tabriz University of Medical Sciences
}

August 28, 2020

\begin{abstract}
Kidney diseases have recently become one of the major global health problems with high incidence and death risk. Extracellular vesicles (EVs), which are released from most cell types and human body fluids, have recently attracted extensive attention as the important carriers of protective cargos such as microRNAs and proteins that may contribute to promoted renal function. Therefore, we conducted this systematic review to investigate the renoprotective potential of EVs. We performed a comprehensive search of Medline (via PubMed), Embase, Cochrane and Proquest on August, 2019 for English papers. Of 2887 articles met the search criteria, 80 included in this systematic review and classified into several groups based on source of EVs. We then evaluated the EV research through screening their isolation method, size, marker, main effector, and potential mechanisms to induce renal protection. Taken together, EVs from various sources including mesenchymal stem cells, human liver stem cells, endothelial progenitor cells, endothelial colony-forming cells, serum and some other sources might have positive impacts on treatment of human kidney diseases in the future.
\end{abstract}

\section{Introduction}

kidney diseases including acute kidney injury (AKI) and chronic kidney disease (CKD), are one of the most important health issues and account for an appreciable morbidity and mortality worldwide ${ }^{1}$. AKI is defined by a rapid (hours to days) deterioration in kidney function and remains a clinical concern impacts approximately $5 \%$ of hospitalized patients ${ }^{2}$. It has been reported that ischemia/reperfusion injury (IRI), sepsis and nephrotoxic drugs are potential causes of this disorder ${ }^{3}$. CKD, which affects $8-16 \%$ of the population in the world, is a progressive process that is related to a high risk of diabetics, hypertension, and cardiovascular disorders and eventually results in end-stage renal disease (ESRD) ${ }^{4,5}$. AKI is also recognized as an independent contributive factor to ESRD, increasing the incident CKD ${ }^{6}$. End-stage CKD is often treated with dialysis or kidney transplantation, which are limited to cost restraints and availability of donor kidneys ${ }^{7}$. Accordingly, exploring potent approaches for prevention and treatment of kidney diseases and regeneration of this tissue is imperative.

In this regard, extracellular vesicles (EVs) have achieved considerable attention as promising renoprotective candidates. EVs are nano-sized vesicles which are secreted by almost all human cell types and act as important mediators of cell-cell communication and many biological process such as tissue repair and regeneration via transferring of cell-specific cargos, including proteins, lipids, miRNAs and RNAs ${ }^{8,9}$. Exosomes are a subtype of EVs (30-150 nm in diameter), which are produced in microvesicular bodies and then secreted into the extracellular space ${ }^{10}$. The next subclass is microvesicles (MVs) which are larger than exosomes (100-1000 $\mathrm{nm}$ in diameter) and are produced via direct budding from the plasma membrane ${ }^{11}$. In comparison with treatments based on whole cells, EVs display a higher safety profile and can be stored without missing function, showing their potential as cell-free therapeutic paradigm ${ }^{8}$. According to the literature, the therapeutic potential of EVs have been revealed in treatment of various kidney diseases ${ }^{12}$. 
To the best of our knowledge, no systematic review article has already been published in this field. Therefore, we conducted this systematic review of the literature to investigate the beneficial effects of EVs and their cargo in protection and treatment of renal diseases.

\section{Methods}

The study protocol was developed according to the Cochrane Collaboration ${ }^{13}$.

\subsection{Criteria for considering studies for this review}

\subsubsection{Types of studies}

All experimental studies which assessed the EVs (exosomes, microvesicles, and apoptotic bodies) in in vitro or in vivo models (animal and human) were included in this review.

\subsubsection{Types of interventions}

All publications which studied the renoprotective potential of EVs or EV-derived components were included.

2.2. Search methods for identification of studies

Databases including MEDLINE via PubMed, Embase, Cochrane library (CENTRAL), and Proquest (for dissertation) were searched for papers from inception to the August 2019, using the identified keywords and index terms. Trials published in English were eligible for this review. Full details of the search strategies for PubMed is reported in Appendix I. The reference lists of all included articles were also checked to retrieve remaining relevant studies.

\subsection{Data collection and analysis}

\subsubsection{Selection of studies}

All identified citations were loaded into EndNote X9 (https://endnote.com) software and duplicate studies were eliminated. Titles and abstracts were screened for inclusion by two individual authors (F.G. and H.A.). Then, the same two authors independently retrieved and evaluated the full text of potentially relevant articles. A third review author (M.T.) was available to achieve an agreement if required. Search results are presented in a PRISMA flow diagram ${ }^{14}$.

\subsubsection{Assessment of risk of bias in included studies}

The quality of the eligible articles was evaluated in duplicate by independent authors (F.G. and H.A.) using an appraisal tool adapted from Wendt et al. (2018) study to clarify EV characteristics ${ }^{9}$. Any unresolved discrepancy was referred to a third author (M.T.).

\subsubsection{Data extraction}

Two reviewers independently (F.G. and H.A.) extracted the following data from the eligible studies: The first author's name, publication year, injury model as well as EV source, isolation method, size, EV-derived cargo as the main effector, investigated markers, and the renoprotective results achieved in each study. In case of doubt, a third reviewer was consulted (M.T.).

\section{Results}

\subsection{Study selection}

The combined database searches yielded a total of 2887 publications including 1026 articles for PubMed, 1739 articles for Embase, 33 article for Cochrane library and 89 article for Proquest. The 986 duplicates were discarded and after screening the title and abstract of the remaining references, 128 related studies were retrieved. Further evaluation of the full text of remaining citations resulted in the inclusion of 80 articles for qualitative assessment in this systematic review. The flowchart of study selection process and number of publications at each stage is depicted in Figure 1. 


\subsection{Quality of EV research}

As summarized in Table 1, the quality assessment of the EV research was evaluated via screening the EV isolation procedure, their associated markers and availability of electron microscopy (EM) pictures. Ultracentrifugation was the most commonly procedure used for the purification and four studies employed precipitation to purify the EVs ${ }^{15-18}$. Of all the publications, 22 studies did not employ EM examination of the isolated EVs and 19 studies did not determine the typical markers of the purified EVs. We further determined if the expressed methods were reported in the results part (reporting bias) and whether any statement about disclosure/conflict of interest might have affected the results (other bias).

\subsection{Characteristics of the included studies}

In the following sections, we summarized the studies matching our search criteria and evaluating the potential effects of EVs originated from different sources in renal protection.

\subsubsection{Mesenchymal stem cells}

Mesenchymal stem cells (MSCs) are self-renewing multipotent progenitors with differentiation ability into various cell types ${ }^{19}$. It has been revealed that the majority of regenerative effects of MSCs are related to their capacity in secretion of EVs as critical mediators of paracrine action ${ }^{20}$. As summarized in Table 2, We have found 48 studies investigated the renoprotective effects of MSC-EVs which are discussed in the following parts.

Bruno and coworkers showed for the first time that mRNA from bone marrow-derived MSC-EVs (BMMSC-EVs) contribute to kidney protection in AKI animal model by stimulating the proliferation of tubular epithelial cells (TECs) and inhibiting apoptosis ${ }^{21}$. Gatti et al. (2011) have also found that BM-MSC-EVs can protect IRI rat model from AKI via reducing apoptosis and increasing TEC proliferation ${ }^{22}$. These EVs also shown to favor kidney repair in experimental model of nephrectomy ${ }^{23}$. Furthermore, BM-MSCs were indicated to have ability to minimize the renal injury through RNAs carried by their EVs ${ }^{24}$. Another in vitro and in vivo experiments also indicated that BM-MSC-EVs exert a pro-survival effect on renal cells by inhibiting apoptosis ${ }^{25}$. Tomasoni et al. (2013) suggested that transfer of growth factor receptor (IGF-1R) mRNA by BM-MSC-EVs favoring renal protection ${ }^{26}$. In another in vitro model of IRI, BMMSC-EVs were enhanced the recovery process in proximal TECs through transferring and modulating of the expression of multiple microRNAs ${ }^{27}$. It has been also evidenced that microRNAs have crucial role in regenerative effects of BM-MSCs in $\mathrm{AKI}^{28}$. He et al. (2015) proposed that EVs isolated from BMMSCs led to the recovery of injured kidney both in in vitroand unilateral ureteral obstruction (UUO) mice model through microRNA-dependent repairing ${ }^{29}$. Moreover, it was reported by Wang and coworkers that BM-MSC-EVs treated with erythropoietin have superior effect in protection against UUO renal injuries in comparison with MSC-EVs ${ }^{30}$. Another research conducted by Nagaishi et al. (2016) proved that intravenous administration of BM-MSC-derived EVs could ameliorate diabetic nephropathy (DN). They showed that these EVs reduced abnormal BM-derived cells infiltration into the kidney, prevented the expression of proinflammatory cytokines and fibrosis, suppressed epithelial-mesenchymal transformation (EMT) of TECs, exerted an anti-apoptotic potential and protected tight junction structure in TECs ${ }^{15}$. Shen et al. (2016) highlighted that the C-C motif chemokine receptor-2 (CCR2) positive EVs secreted by BM-MSCs can play as decoys to suppress CCL2 activity and subsequently result in inhibition of macrophage activation and IRI alleviation ${ }^{31}$. Engineered BM-MSCs deliver miR-let7 via EVs were also confirmed to have anti-fibrotic effects in kidney ${ }^{16}$. It has been observed that exosomes originated from BM-MSCs have more proliferative potentials to promote AKI recovery in comparison with microvesicles ${ }^{32}$. Additionally, the activity of different sub-populations of EVs isolated from BM-MSCs using the gradient separation technique, was analyzed on renal TECs ${ }^{33}$. The authors indicated that the medium-density CF2 fraction enriched in exosomes had the best activity on renal TECs proliferation and inhibition of apoptosis. Wang and coworkers have revealed that miR-199a-5p from BM-MSC-EVs can protect against renal IRI in vitro and in vivo through significant suppressing of endoplasmic reticulum stress ${ }^{34}$. Similarly, Zhu et al. (2019) have recently showed that miR-199a-3p released from BM-MSC-EVs can protect against IRI via inducing anti-apoptotic effect ${ }^{35}$. BM- 
MSCs derived EVs can also contribute to renoprotection in an animal model of DN through their anti-fibrotic potential and by autophagy induction related to the down-regulation of mTOR ${ }^{36}$. A similar animal study also demonstrated that these EVs could attenuate DN via preventing fibrosis ${ }^{37}$. Tapparo et al. (2019) have additionally postulated that BM-MSC-EVs engineered with miRNAs are more effective than EVs originated from naïve MSCs in renal regeneration of AKI model ${ }^{38}$. A recent study also found that melatonin can improve therapeutic potential of BM-MSC-EVs against IRI in rat models ${ }^{39}$.

Zhou et al. (2013) indicated that EVs from human umbilical cord-derived MSCs (HUC-MSC-EVs) protect against cisplatin-induced AKI via enhancing oxidative stress and apoptosis, and improving proliferation in vivo and in vitro ${ }^{40}$. Another research by Ju et al. (2015) showed that HUC-MSC-EV-induced hepatocyte growth factor (HGF) can facilitate TEC dedifferentiation and proliferation ${ }^{41}$. Administration of these EVs have also yielded promising results in alleviating kidney injury in IRI rat model via promoting angiogenesis effects in HIF- $1 \alpha$ independent manner ${ }^{42}$. Moreover, another study elucidated that HUC-MSC-EVs may ameliorate renal IRI by down-regulating NK cells by spleen independent manner ${ }^{43}$. In 2016, Nassar et al. conducted the first clinical trial to investigate the safety and therapeutic potential of HUC-MSC-EVs in treatment of grade III \& IV CKD patients. administration of the EVs was safe and ameliorated the inflammatory reaction and enhanced the overall kidney function in the patients ${ }^{44}$. The beneficial therapeutic effects of HUC-MSC-EVs have been also shown in DN mice model ${ }^{45}$. The authors reported that miR-451a shuttled by these vesicles could successfully ameliorate DN by improving EMT and regulating cell cycle via targeting p15 and p19. Additionally, three studies have found that 14-3-3 $\zeta$ delivered by HUC-MSCEVs may prevent the cisplatin-induced nephrotoxicity by activation of autophagy ${ }^{46-48}$. Li et al. (2019), tested the protective effect of HUC-MSC-EVs against oxidative stress and EMT induced by oxalate and calcium oxalate monohydrate crystals in human renal TECs. The results suggested that these EVs reduce the oxidative damage and EMT of proximal tubular epithelial (HK-2) cells that may associated with the up-regulation of TGF- $\beta^{49}$.

As shown in Table 2, six other studies examined the beneficial of EVs from adipose-derived MSCs (AD-MSCs) in this field. For instance, Lin et al. (2016) indicated that combined AD-MSCs and AD-MSCs-derived EVs therapy have superior renoprotective properties in IRI rat models than EVs alone ${ }^{50}$. Moreover, AD-MSCs derived EVs can prevent AKI-CKD transition by TEC dependent Sox9 activation in in vitro and IRI mice models ${ }^{51}$. Eirin et al. (2017) established that intrarenal delivery of EVs derived from autologous AD-MSCs led to the elevation of interlukin-10 (IL-10) and number of reparative macrophages, reduction of renal fibrosis, and thereby ameliorated renal injury in pig model of renal artery stenosis (RAS) complicated by metabolic syndrome (MetS) ${ }^{52}$. A similar study was also confirmed the renoprotective potential of AD-MSC-EVs in swine model of renovascular disease (RVD) + MetS. The results showed that these EVs could ameliorate renal injury through promoting angiogenesis and microvascular repair ${ }^{53}$. Additionally, healthy AD-MSCderived EVs were shown to have superior ability in suppressing inflammatory responses and preventing renal damage in rats with sepsis-induced kidney injury in comparison with apoptotic AD-MSC-EVs ${ }^{54}$. A recent study has also indicated the remarkable ability of hypoxia preconditioned AD-MSC-EVs in improvement of IRI ${ }^{55}$.

The renoprotective ability of EVs from human Wharton's Jelly MSCs (HWJ-MSC-EVs) was also explored in seven studies. Intravenous administration of EVs isolated from HWJ-MSCs immediately after unilateral IRI improved kidney function and reduced oxidative stress, apoptosis, and fibrosis ${ }^{56}$. Similarly, the EVs was able to ameliorate IRI in rats through suppression of CX3CL1 ${ }^{57}$. Moreover, two other studies have found that HWJ-MSC-EVs can recover IRI-induced AKI via decreasing oxidative stress through activating Nrf2/antioxidant response element and inhibition of mitochondrial fission ${ }^{58,59}$. Chen et al. (2017) further supported this notion by indicating the ability of HWJ-MSC-EVs in protection against IRI-induced renal fibrosis ${ }^{60}$. Injection of these EVs immediately post renal transplantation can also significantly ameliorate IRI via enhancing proliferation and decreasing inflammation, apoptosis and renal fibrosis ${ }^{61}$. According to Zhang et al. (2019), HWJ-MSC-EVs have also ability to ameliorate cyclosporin A-induced renal fibrosis in rats through their anti-oxidative properties. 
EVs isolated from kidney-derived MSCs (KMSCs) significantly improved renal function in mouse model of IRI and stimulated proliferation and inhibited apoptosis in vitro ${ }^{62}$. Another study by Choi et al. (2015) also evaluated the renoprotective effects of KMSC-EVs in UUO murine model. Findings revealed that the EVs can improve the real function by rarefaction of peritubular capillaries through inhibition of endothelialto-mesenchymal transition, their anti-fibrotic effects, and ameliorating inflammation ${ }^{62}$.

Ranghino et al. (2017) determined that administration of MSC-EVs within the glomeruli could improve renal function and decrease the ischemic damage after IRI through activation of TEC proliferation. Furthermore, Yuan et al. (2017) reported the anti-necroptosis potential of human-induced pluripotent stem cell-derived MSC-EVs against IRI in vitro and in vivo.

\subsubsection{Tubular epithelial cells}

TECs are the most abundant cell population in the kidney, and carry out various regulatory functions in both physiological and pathological situations ${ }^{63}$. To the best of our knowledge, 10 research articles concerning TEC-derived EVs and kidney diseases have been published so far (Table 3).

Intravenous administration of renal TEC-EVs was significantly enhanced renal function after IRI in rats ${ }^{64}$. The TEC-derived EVs also play a critical role in preventing damage in hypoxic AKI model ${ }^{65}$. Zhang and coworkers additionally supported the therapeutic effects of EVs derived from hypoxic preconditioned HK-2 cells in kidney IRI ${ }^{66}$. More recently, it was reported by Zhang et al. (2019) that hypoxia induced renal TEC-EVs protected against IRI potentially through different EV-microRNAs ${ }^{67}$.

In contrast, EVs containing microRNA-21 secreted from injured TECs promoted tubular phenotype transition in renal fibrosis via targeting depression of phosphatase and tensin homolog (PTEN) protein ${ }^{68}$. Two recent studies have also noticed that microRNA-21- and microRNA-216a-containing EVs from proximal TECs participate in renal interstitial fibrosis by enhancing PTEN/AKT signaling pathway ${ }^{69,70}$. miRNA-23aand miR-19b-3p-enriched EVs from TECs have also shown to promote tubulointerstitial inflammation (TI) through activation of macrophages ${ }^{71,72}$. Recently, García-Pastor et al. (2019) proposed that EVs released from cisplatin-treated proximal TECs could inhibit proliferation and induce apoptosis in EV-recipient HK-2 cells. Interestingly, second generation of EVs secreted by the EV-recipient HK-2 cells, enhanced proliferation of naïve HK-2 cells ${ }^{73}$.

\subsubsection{Human liver stem cells}

Three other articles have investigated the role of human liver stem cells derived EVs (HLSC-EVs) in this context (Table 4).

A study conducted by Sanchez et al. in 2014, has examined the effect of HLSC-EVs in promotion of tubular regeneration post AKI. In vivofindings showed significant improvement in renal function and morphology. In vitro experiments also indicated their capacity in stimulating proliferation and inhibition of apoptosis ${ }^{74}$. In another study, the authors demonstrated the regenerative, anti-fibrotic, and anti-inflammatory impact of HLSC-derived EVs in an aristolochic acid nephropathy (AAN) mouse model of CKD ${ }^{75}$. A recent animal study has also acknowledged that HLSC-EVs enriched in microRNAs can inhibit fibrosis and its progression in $\mathrm{DN}^{37}$.

\subsubsection{Endothelial progenitor cells}

$\mathrm{BM}$-derived endothelial progenitor cells (EPCs) are circulating precursors possess the ability to migrate to the areas of vascular injury and induce regeneration ${ }^{76}$. We found three publications have studied the EPC-EVs protective effects on renal diseases (Table 5).

In an experimental model of IRI, intravenous injection of EPC-EVs exerted a microRNA mediated protective effect through enhancing tubular cell proliferation, inhibiting apoptosis and leukocyte

infiltration ${ }^{77}$. In a similar work, Cantaluppi et al. (2015) documented that EVs released by EPCs could protect against antibody/complement-mediated injury of mesangial cells in Thy1.1 glomerulonephritis ${ }^{78}$. A 
recent study has also highlighted the efficiency of putative EPC-EVs in reducing UUO-induced renal fibrosis via inhibiting pericyte-myofibroblast transition without significant promotion of vascular repair ${ }^{79}$.

\subsubsection{Endothelial colony-forming cells}

Endothelial colony-forming cells (ECFCs) are a population of EPCs that have displayed considerable therapeutic benefits due to their high proliferative potential and vessel-forming capacity ${ }^{80}$. Three studies are also assessed the renoprotective effects of EVs from endothelial colony-forming cells (ECFCs) (Table 6).

Burger and coworkers found the protective effects of human umbilical cord blood-derived ECFC-EVs (exosomes, but not microparticles) in IRI mouse model. In vitro experiments also indicated that exosomes are the main mediator of endothelial protection from apoptosis ${ }^{81}$. Moreover, Vinas et al. (2016) has reported microRNA-486-5p transferred by ECFC-EVs as an effective molecule in renoprotection in mouse with IRI related to their ability in reducing kidney PTEN ${ }^{82}$. In another study performed by Vinas et al. (2018), the ECFC-EVs was shown to selectively target the kidneys post IRI in which exosomal CXC chemokine receptor type 4 (CXCR4) interaction with stromal cell-derived factor (SDF)-1 $\alpha$ was known as a critical process for transferring of microRNA-486-5 $\mathrm{p}^{83}$.

\subsubsection{Fibroblast}

In 2014, Zhou et al. showed that miR-34a-containing EVs originated from fibroblasts contribute to tubular cell apoptosis in UUO-induced fibrotic kidney ${ }^{84}$. Similarly, impaired renal interstitial fibroblast-derived EVs containing miR-34a promoted TEC apoptosis and involved in renal interstitial fibrosis through suppressing Bcl-2 expression ${ }^{85}$. Bruno et al. (2012) postulated that EVs from fibroblasts couldn't prevent TEC apoptosis in lethal model of $\mathrm{AKI}^{25}$. Furthermore, two other studies indicated that fibroblast EVs had no protective impact on kidney ${ }^{40,77}$. Grange et al. (2019) also reported that fibroblast derived EVs provided no antifibrotic effects in a mouse model of $\mathrm{DN}^{37}$ (Table 7).

\subsubsection{Platelets}

The pathogenic role of Platelet-derived EVs have been recently demonstrated in glomerular endothelial injury in early DN via secretion of CXCL7 ${ }^{86}$. Additionally, Wu et al. (2019) proposed that miR-191 released from platelet-EVs induced apoptosis of TECs and involved in development of IRI by inhibiting Cystathionine- $\beta$-synthase $^{87}$ (Table 8).

\subsubsection{Serum}

Zhang et al. (2017) suggested that the underlying mechanism of remote ischemic preconditioning (rIPC) in alleviating kidney IRI is related to their ability in inducing TECs to release functional EVs to the serum ${ }^{66}$. It has been also shown that limb rIPC protect mouse renal from septic AKI by elevating miR-21 in EV of mouse serum ${ }^{88}$ (Table 9).

\subsubsection{Other sources}

We have found several other articles studied the benefits of EVs from other sources in kidney diseases (Table 10). For instance, epithelial cell-derived EVs containing ATF3 RNA reduces IRI by down-regulating pro-inflammatory gene MCP-1 ${ }^{89}$. Moreover, TGF- $\beta 1$-contaning EVs derived from high glucose-treated glomerular endothelial cells promoted renal fibrosis in DN model through activation of glomerular mesangial cells ${ }^{18}$. Jiang et al. (2016) reported that EVs from urine-derived stem cells could prevent renal injury in rats with type I diabetic which may be associated with reducing apoptosis in podocyte, enhancing angiogenesis and cell survival ${ }^{90}$. Furthermore, the results of a recent animal study indicated that miR-486 enriched in adipose-derived stem cell-EVs have the potential to attenuate DN by inhibiting podocyte apoptosis and activating autophagy ${ }^{91}$. It has also been reported that EVs secreted by renal scattered tubular-like cells contribute to repairing damaged TECs in vitro and in vivo by their mitochondria cargo ${ }^{92}$. In another UUO mice model, engineered exosomes enriched in miR-29 attenuated renal fibrosis by suppressing Yin Yang 1 (YY1) and TGF- $\beta$ pathway proteins ${ }^{93}$. Pan et al. (2019) also showed that EVs enriched in miR-21 derived 
from cultured myotubes with $\mathrm{H} / \mathrm{R}$, exert anti-inflammatory and anti-apoptotic effects and subsequently attenuate sepsis-induced AKIin vitro ${ }^{88}$.

However, according to the study conducted by Martínez et al. (2014), EVs released by vascular endothelial cells might have either beneficial or harmful properties in different renal diseases through activation of HIF- $\alpha$ and VEGF-A signaling ${ }^{94}$. EVs from T-CD $133^{+}$cells (renal tubular CD133 ${ }^{+}$progenitor cells) were shown to have no significant contribution in renal recovery after IRI ${ }^{95}$. It has been also evidenced that podocyte-EVs induce fibrosis in renal proximal TECs by activation of p38 MAPK and CD36 ${ }^{96}$. Furthermore, multiple myeloma cell-EVs play potential role in apoptosis of HK-2 cells and led to renal impairment in these patients 97.

\section{Discussion}

In this systematic review we overviewed the renoprotective properties of EVs for the first time. Overall the results provide evidence in support of beneficial properties of EVs from various sources in prevention or treatment of kidney disorders. However, some EVs secreted by TECs ${ }^{68-73}$, fibroblasts ${ }^{84,85}$, platelets ${ }^{86,87}$, podocyte ${ }^{96}$, and MM cells ${ }^{97}$ were found to have adverse effects on renal function. Five other studies also suggested that EVs from fibroblasts ${ }^{25,37,40,77}$ and T-CD133 ${ }^{+}$cells $^{95}$ may have no beneficial effects on renal injury protection.

Increased number of studies have revealed that MSCs are one of the best candidates to treat human diseases through their capacity in secretion of EVs ${ }^{8}$. According to the literature of this review, MSCs, especially BM-derived MSCs, are the most abundant type of employed EV sources in protection of kidney injuries.

In the present systematic review, preclinical studies established different models of acute or chronic kidney injuries induced by IRI, DN, UUO, and nephrotoxicity as well as RAS, RVD, sepsis, and TI. Despite all the beneficial advantages of EV therapy in various kidney diseases, we have identified only one clinical trial in this field. According to the literature, EV therapy in most of the cases successfully enhanced renal function through decreasing apoptosis and elevating proliferation of TECs, suppressing inflammatory responses, reducing oxidative stress and fibrosis, and promoting angiogenesis.

Most of these renoprotective effects were mediated by EV-derived microRNAs. For instance, miR-let7c, miR451a and miR-29 contributed to renal function enhancement through reduction of fibrosis, whereas miR-21, miR-216a and miR-34a participated in induction of fibrosis. Besides, miR-199a-5p, miR-30, miR-21, miR486-5p and miR-451a were shown to have anti-apoptotic or proliferative effects on renal cells, however, miR-191 and miR-34a induced apoptosis. Some other microRNAs such as miR-23a and miR-19b-3p could also up-regulate inflammation in kidney diseases. Additionally, miR-21 was able to play renoprotective role via suppression of inflammatory responses. EVs engineered to alter their microRNA contents revealed to have potential renoprotective properties. It has been also shown that reduction of renal tubular cell apoptosis

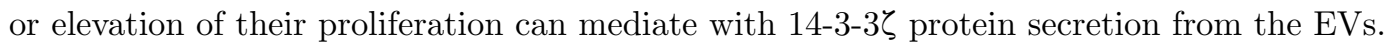

In spite of its beneficial role in renal injury enhancement, EV therapy may increase the risk for developing renal cancer. For instance, EVs released from HWJ-MSCs promoted renal cancer cell growth and invasiveness by inducing hepatocyte growth factor (Du et al., 2014). In two separate studies, the role of EVs originated from 786-0 renal cancer cell line was determined in renal tumor development in vitro ${ }^{98,99}$. They reported that application of these EVs result in renal cancer progression by promoting angiogenesis via suppressing the hepatocyte cell adhesion molecule (hepaCAM), 786-0 cell migration and invasion. Similarly, Jiang and coworkers suggested EVs released by renal carcinoma OS-RC-2 cell line contribute to the suppression of hepaCAM expression ${ }^{100}$. The impact of renal cancer stem cell (CSC)-derived EVs was also investigated in phenotypical changes of MSCs which favor tumor progression by Lindoso et al. (2015). The data indicated that the EV-stimulated MSCs can support tumor progression and vascularization both in in vitro andin vivo models. In another study, the impact of clear cell renal cell carcinoma (CCRCC) derived CSC-EVs in the EMT progression and lung metastasis of CCRCC cells was explored. The findings suggested that CD103 ${ }^{+}$CSC-EVs can induce EMT promoting metastasis via transferring of miR-19b-3p ${ }^{101}$. In contrast, the antitumor potential of IL-12-anchored renal cancer cell-derived EVs was observed in renal cell carcinoma 
by Zhang et al (2010).

Finally, there are several limitations pertinent to this systematic review. First, due to the heterogeneity in design and methods evaluating the outcomes in the included publications, conducting a meta-analysis was not possible. Furthermore, we failed to contact the authors of abstract articles and were able to include only studies in English. It is also important to acknowledge that systematic reviews of preclinical studies may present a bias towards an overestimation of positive outcomes since negative results may be less often published and no information concerning safety is available.

\section{Conclusion}

Taken together, this systematic review will be the first to provide an estimate on efficacy of EV therapy in in vitro and in vivokidney injury models. Although EVs from several sources were shown to have adverse impacts on renal protection, the present systematic review underlined that EVs might represent a promising therapeutic strategy in treatment of kidney diseases. In addition, future work should aim to better design of preclinical studies to improve the safety evaluation of the EV therapy before proceeding to human subjects.

\section{Acknowledgment}

The present research was done with the support of Student research committee, Tabriz University of Medical Sciences, Tabriz, Iran.

\section{Funding information}

Student research committee, Tabriz University of Medical Sciences, Tabriz, Iran, Grant/Award Number: 63597

\section{Conflict of interest}

Authors have no conflict of interests.

Author Contribution : Hossein Abbaszadeh and Farzaneh Ghorbani conducted the search and screening of titles, abstracts, full-text articles, the study selection, data extraction and quality, screened the full-text articles, and assessed the eligibility of the studies and contributed to the writing of the manuscript. Aliakbar Movassaghpour, Mehdi Talebi and Mehdi Yousefi designed the study protocol, wrote some parts of the manuscript and edited the final version. All authors read the final version of the manuscript.

\section{References}

1. Yun WC, Lee HS. Potential and Therapeutic Efficacy of Cell-based Therapy Using Mesenchymal Stem Cells for Acute/chronic Kidney Disease.International Journal of Molecular Sciences. 2019;20(7).

2. Viñas JL, Spence M, Gutsol A, et al. Receptor-Ligand Interaction Mediates Targeting of Endothelial Colony Forming Cell-derived Exosomes to the Kidney after Ischemic Injury. Scientific reports.2018;8(1):16320.

3. Negi S, Koreeda D, Kobayashi S, et al. Acute kidney injury: Epidemiology, outcomes, complications, and therapeutic strategies. Seminars in dialysis. 2018;31(5):519-527.

4. Mullins LJ, Conway BR, Menzies RI, Denby L, Mullins JJ. Renal disease pathophysiology and treatment: contributions from the rat. Dis Model Mech. 2016;9(12):1419-1433.

5. Nugent RA, Fathima SF, Feigl AB, Chyung D. The burden of chronic kidney disease on developing nations: a 21st century challenge in global health. Nephron Clinical practice. 2011;118(3):c269-277.

6. Chawla LS, Kimmel PL. Acute kidney injury and chronic kidney disease: an integrated clinical syndrome. Kidney international.2012;82(5):516-524.

7. Sun D, Bu L, Liu C, et al. Therapeutic effects of human amniotic fluid-derived stem cells on renal interstitial fibrosis in a murine model of unilateral ureteral obstruction. PloS one.2013;8(5):e65042. 
8. Abbaszadeh H, Ghorbani F, Derakhshani M, Movassaghpour A, Yousefi M. Human umbilical cord mesenchymal stem cell-derived extracellular vesicles: A novel therapeutic paradigm. Journal of cellular physiology. 2019 .

9. Wendt S, Goetzenich A, Goettsch C, et al. Evaluation of the cardioprotective potential of extracellular vesicles - a systematic review and meta-analysis. Scientific Reports. 2018;8(1):15702.

10. Kalra H, Drummen GP, Mathivanan S. Focus on Extracellular Vesicles: Introducing the Next Small Big Thing. Int J Mol Sci.2016;17(2):170.

11. Borges FT, Reis LA, Schor N. Extracellular vesicles: structure, function, and potential clinical uses in renal diseases. Braz J Med Biol Res. 2013;46(10):824-830.

12. Shao K, Wang B, Nie A, et al. Target-triggered signal-on ratiometric electrochemiluminescence sensing of PSA based on MOF/Au/G-quadruplex.Biosensors \& bioelectronics. 2018;118:160-166.

13. Higgins JP, Altman DG, Gøtzsche PC, et al. The Cochrane Collaboration's tool for assessing risk of bias in randomised trials. 2011;343:d5928.

14. Moher D, Liberati A, Tetzlaff J, Altman DG, The PG. Preferred Reporting Items for Systematic Reviews and Meta-Analyses: The PRISMA Statement. PLOS Medicine. 2009;6(7):e1000097.

15. Nagaishi K, Mizue Y, Chikenji T, et al. Mesenchymal stem cell therapy ameliorates diabetic nephropathy via the paracrine effect of renal trophic factors including exosomes. Scientific reports.2016;6:34842.

16. Wang B, Yao K, Huuskes BM, et al. Mesenchymal stem cells deliver exogenous MicroRNA-let7c via exosomes to attenuate renal fibrosis. Molecular Therapy. 2016;24(7):1290-1301.

17. Yuan X, Li D, Chen X, et al. Extracellular vesicles from human-induced pluripotent stem cell-derived mesenchymal stromal cells (hiPSC-MSCs) protect against renal ischemia/reperfusion injury via delivering specificity protein (SP1) and transcriptional activating of sphingosine kinase 1 and inhibiting necroptosis. Cell Death and Disease. 2017;8(12).

18. Wu X, Gao Y, Xu L, et al. Exosomes from high glucose-treated glomerular endothelial cells trigger the epithelial-mesenchymal transition and dysfunction of podocytes. Scientific reports.2017;7(1):9371.

19. Freisinger E, Cramer C, Xia X, et al. Characterization of hematopoietic potential of mesenchymal stem cells. Journal of Cellular Physiology. 2010;225(3):888-897.

20. Jing H, He X, Zheng J. Exosomes and regenerative medicine: state of the art and perspectives. Translational research : the journal of laboratory and clinical medicine. 2018;196:1-16.

21. Bruno S, Grange C, Deregibus MC, et al. Mesenchymal stem cell-derived microvesicles protect against acute tubular injury. Journal of the American Society of Nephrology.2009;20(5):1053-1067.

22. Gatti S, Bruno S, Deregibus MC, et al. Microvesicles derived from human adult mesenchymal stem cells protect against ischaemia-reperfusion-induced acute and chronic kidney injury.Nephrology Dialysis Transplantation. 2011;26(5):1474-1483.

23. He J, Wang Y, Sun S, et al. Bone marrow stem cells-derived microvesicles protect against renal injury in the mouse remnant kidney model. Nephrology (Carlton). 2012;17(5):493-500.

24. Reis LA, Borges FT, Simoes MJ, Borges AA, Sinigaglia-Coimbra R, Schor N. Bone marrow-derived mesenchymal stem cells repaired but did not prevent gentamicin-induced acute kidney injury through paracrine effects in rats. PLoS One. 2012;7(9):e44092.

25. Bruno S, Grange C, Collino F, et al. Microvesicles derived from mesenchymal stem cells enhance survival in a lethal model of acute kidney injury. PLoS ONE. 2012;7(3). 
26. Tomasoni S, Longaretti L, Rota C, et al. Transfer of growth factor receptor mRNA via exosomes unravels the regenerative effect of mesenchymal stem cells. Stem cells and development.2013;22(5):772-780.

27. Lindoso RS, Collino F, Bruno S, et al. Extracellular vesicles released from mesenchymal stromal cells modulate miRNA in renal tubular cells and inhibit ATP depletion injury. Stem Cells and Development. 2014;23(15):1809-1819.

28. Collino F, Bruno S, Incarnato D, et al. AKI recovery induced by mesenchymal stromal cell- derived extracellular vesicles carrying micrornas. Journal of the American Society of Nephrology.2015;26(10):23492360 .

29. He J, Wang Y, Lu X, et al. Micro-vesicles derived from bone marrow stem cells protect the kidney both in vivo and in vitro by microRNA-dependent repairing. Nephrology. 2015;20(9):591-600.

30. Wang Y, Lu X, He J, Zhao W. Influence of erythropoietin on microvesicles derived from mesenchymal stem cells protecting renal function of chronic kidney disease. Stem Cell Research and Therapy. 2015;6(1).

31. Shen B, Liu J, Zhang F, et al. CCR2 Positive Exosome Released by Mesenchymal Stem Cells Suppresses Macrophage Functions and Alleviates Ischemia/Reperfusion-Induced Renal Injury. Stem Cells International. $2016 ; 2016$.

32. Bruno S, Tapparo M, Collino F, et al. Renal Regenerative Potential of Different Extracellular Vesicle Populations Derived from Bone Marrow Mesenchymal Stromal Cells. Tissue Engineering - Part A.2017;23(2122):1262-1273.

33. Collino F, Pomatto M, Bruno S, et al. Exosome and Microvesicle-Enriched Fractions Isolated from Mesenchymal Stem Cells by Gradient Separation Showed Different Molecular Signatures and Functions on Renal Tubular Epithelial Cells. Stem Cell Reviews and Reports.2017;13(2):226-243.

34. Wang C, Zhu G, He W, et al. BMSCs protect against renal ischemia-reperfusion injury by secreting exosomes loaded with miR-199a-5p that target BIP to inhibit endoplasmic reticulum stress at the very early reperfusion stages. FASEB journal : official publication of the Federation of American Societies for Experimental Biology. 2019;33(4):5440-5456.

35. Zhu G, Pei L, Lin F, et al. Exosomes from human-bone-marrow-derived mesenchymal stem cells protect against renal ischemia/reperfusion injury via transferring miR-199a-3p. J Cell Physiol.2019;234(12):2373623749 .

36. Ebrahim N, Ahmed IA, Hussien NI, et al. Mesenchymal Stem Cell-Derived Exosomes Ameliorated Diabetic Nephropathy by Autophagy Induction through the mTOR Signaling Pathway. Cells. 2018;7(12).

37. Grange C, Tritta S, Tapparo M, et al. Stem cell-derived extracellular vesicles inhibit and revert fibrosis progression in a mouse model of diabetic nephropathy. Scientific Reports.2019;9(1):4468.

38. Tapparo M, Bruno S, Collino F, et al. Renal regenerative potential of extracellular vesicles derived from mirna-engineered mesenchymal stromal cells. International Journal of Molecular Sciences.2019;20(10).

39. Alzahrani FA. Melatonin improves therapeutic potential of mesenchymal stem cells-derived exosomes against renal ischemia-reperfusion injury in rats. American Journal of Translational Research. 2019;11(5):2887-2907.

40. Zhou Y, Xu H, Xu W, et al. Exosomes released by human umbilical cord mesenchymal stem cells protect against cisplatin-induced renal oxidative stress and apoptosis in vivo and in vitro. Stem Cell Research and Therapy. 2013;4(2).

41. Ju GQ, Cheng J, Zhong L, et al. Microvesicles derived from human umbilical cord mesenchymal stem cells facilitate tubular epithelial cell dedifferentiation and growth via hepatocyte growth factor induction. PloS one. 2015;10(3):e0121534. 
42. Zou X, Gu D, Xing X, et al. Human mesenchymal stromal cell-derived extracellular vesicles alleviate renal ischemic reperfusion injury and enhance angiogenesis in rats. American Journal of Translational Research. 2016;8(10):4289-4299.

43. Zou X, Gu D, Zhang G, et al. NK cell regulatory property is involved in the protective role of MSC-derived extracellular vesicles in renal ischemic reperfusion injury. Human Gene Therapy.2016;27(11):926-935.

44. Nassar W, El-Ansary M, Sabry D, et al. Umbilical cord mesenchymal stem cells derived extracellular vesicles can safely ameliorate the progression of chronic kidney diseases. Biomater Res.2016;20:21-21.

45. Zhong L, Liao G, Wang X, et al. Mesenchymal stem cells-microvesicle-miR-451a ameliorate early diabetic kidney injury by negative regulation of P15 and P19. Experimental Biology and Medicine. 2018;243(1516):1233-1242.

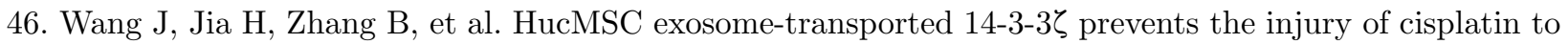
HK-2 cells by inducing autophagy in vitro. Cytotherapy. 2018;20(1):29-44.

47. Jia H, Liu W, Zhang B, et al. HucMSC exosomes-delivered 14-3-3 enhanced autophagy via modulation of ATG16L in preventing cisplatin-induced acute kidney injury. Am J Transl Res.2018;10(1):101-113.

48. Wang B, Jia H, Zhang B, et al. Pre-incubation with hucMSC-exosomes prevents cisplatin-induced nephrotoxicity by activating autophagy.Stem Cell Research and Therapy. 2017;8(1).

49. Li D, Zhang D, Tang B, et al. Exosomes from human umbilical cord mesenchymal stem cells reduce damage from oxidative stress and the epithelial-mesenchymal transition in renal epithelial cells exposed to oxalate and calcium oxalate monohydrate. Stem Cells International. 2019;2019.

50. Lin KC, Yip HK, Shao PL, et al. Combination of adipose-derived mesenchymal stem cells (ADMSC) and ADMSC-derived exosomes for protecting kidney from acute ischemia-reperfusion injury. Int $J$ Cardiol.2016;216:173-185.

51. Zhu F, Chong Lee Shin OLS, Pei G, et al. Adipose-derived mesenchymal stem cells employed exosomes to attenuate AKI-CKD transition through tubular epithelial cell dependent Sox9 activation. Oncotarget.2017;8(41):70707-70726.

52. Eirin A, Zhu XY, Puranik AS, et al. Mesenchymal stem cell-derived extracellular vesicles attenuate kidney inflammation. Kidney Int.2017;92(1):114-124.

53. Eirin A, Zhu XY, Jonnada S, Lerman A, van Wijnen AJ, Lerman LO. Mesenchymal Stem Cell-Derived Extracellular Vesicles Improve the Renal Microvasculature in Metabolic Renovascular Disease in Swine. Cell Transplantation. 2018;27(7):1080-1095.

54. Chang CL, Sung PH, Chen KH, et al. Adipose-derived mesenchymal stem cell-derived exosomes alleviate overwhelming systemic inflammatory reaction and organ damage and improve outcome in rat sepsis syndrome.American Journal of Translational Research. 2018;10(4):1053-1070.

55. Collino F, Lopes JA, Corrêa S, et al. Adipose-Derived Mesenchymal Stromal Cells Under Hypoxia: Changes in Extracellular Vesicles Secretion and Improvement of Renal Recovery after Ischemic Injury. Cellular physiology and biochemistry : international journal of experimental cellular physiology, biochemistry, and pharmacology.2019;52(6):1463-1483.

56. Zhang G, Zou X, Miao S, et al. The anti-oxidative role of micro-vesicles derived from human WhartonJelly mesenchymal stromal cells through NOX2/gp91(phox) suppression in alleviating renal ischemiareperfusion injury in rats. PLoS One. 2014;9(3):e92129.

57. Zou X, Zhang G, Cheng Z, et al. Microvesicles derived from human Wharton's Jelly mesenchymal stromal cells ameliorate renal ischemia-reperfusion injury in rats by suppressing CX3CL1. Stem Cell Research and Therapy. 2014;5(2). 
58. Zhang G, Zou X, Huang Y, et al. Mesenchymal stromal cell-derived extracellular vesicles protect against acute kidney injury through anti-oxidation by enhancing Nrf2/ARE activation in rats. Kidney and Blood Pressure Research. 2016;41(2):119-128.

59. Gu D, Zou X, Ju G, Zhang G, Bao E, Zhu Y. Mesenchymal Stromal Cells Derived Extracellular Vesicles Ameliorate Acute Renal Ischemia Reperfusion Injury by Inhibition of Mitochondrial Fission through MIR-30. Stem Cells International. 2016;2016.

60. Chen W, Yan Y, Song C, Ding Y, Du T. Microvesicles derived from human Wharton's Jelly mesenchymal stem cells ameliorate ischemia-reperfusion-induced renal fibrosis by releasing from G2/M cell cycle arrest. Biochemical Journal. 2017;474(24):4207-4218.

61. Wu X, Yan T, Wang Z, et al. Micro-vesicles derived from human Wharton's Jelly mesenchymal stromal cells mitigate renal ischemia-reperfusion injury in rats after cardiac death renal transplantation. Journal of Cellular Biochemistry.2018;119(2):1879-1888.

62. Choi HY, Lee HG, Kim BS, et al. Mesenchymal stem cell-derived microparticles ameliorate peritubular capillary rarefaction via inhibition of endothelial-mesenchymal transition and decrease tubulointerstitial fibrosis in unilateral ureteral obstruction.Stem Cell Research and Therapy. 2015;6(1).

63. Lv L-L, Feng Y, Tang T-T, Liu B-C. New insight into the role of extracellular vesicles in kidney disease. J Cell Mol Med.2019;23(2):731-739.

64. Dominguez JH, Liu Y, Gao H, Dominguez JM, Xie D, Kelly KJ. Renal tubular cell-derived extracellular vesicles accelerate the recovery of established renal ischemia reperfusion injury. Journal of the American Society of Nephrology. 2017;28(12):3533-3544.

65. Dominguez JM, Dominguez JH, Xie D, Kelly KJ. Human extracellular microvesicles from renal tubules reverse kidney ischemia-reperfusion injury in rats. PloS one. 2018;13(8):e0202550.

66. Zhang G, Yang Y, Huang Y, et al. Hypoxia-induced extracellular vesicles mediate protection of remote ischemic preconditioning for renal ischemia-reperfusion injury. Biomedicine and Pharmacotherapy.2017;90:473-478.

67. Zhang L, Liu H, Xu K, et al. Hypoxia preconditioned renal tubular epithelial cell-derived extracellular vesicles alleviate renal ischaemia-reperfusion injury mediated by the HIF-1alpha/Rab22 pathway and potentially affected by microRNAs. Int J Biol Sci.2019;15(6):1161-1176.

68. Zhou Y, Xiong M, Fang L, et al. MiR-21-containing microvesicles from injured tubular epithelial cells promote tubular phenotype transition by targeting PTEN protein. American Journal of Pathology.2013;183(4):1183-1196.

69. Zheng SB, Zheng Y, Jin LW, Zhou ZH, Li ZY. Microvesicles containing microRNA-21 secreted by proximal tubular epithelial cells are involved in renal interstitial fibrosis by activating AKT pathway. European Review for Medical and Pharmacological Sciences. 2018;22(3):707-714.

70. Qu NY, Zhang ZH, Zhang XX, Xie WW, Niu XQ. Microvesicles containing microRNA-216a secreted by tubular epithelial cells participate in renal interstitial fibrosis through activating PTEN/AKT pathway.European Review for Medical and Pharmacological Sciences.2019;12(15):6629-6636.

71. Li ZL, Lv LL, Tang TT, et al. HIF-1 $\alpha$ inducing exosomal microRNA-23a expression mediates the cross-talk between tubular epithelial cells and macrophages in tubulointerstitial inflammation. Kidney International. 2019;95(2):388-404.

72. Lv LL, Feng Y, Wu M, et al. Exosomal miRNA-19b-3p of tubular epithelial cells promotes M1 macrophage activation in kidney injury. Cell Death and Differentiation. 2019.

73. Garcia-Pastor C, Blazquez-Serra R, Bosch RJ, Lucio Cazana FJ, Fernandez-Martinez AB. Apoptosis and cell proliferation in proximal tubular cells exposed to apoptotic bodies. Novel pathophysiological implications 
in cisplatin-induced renal injury. Biochimica et biophysica acta Molecular basis of disease. 2019;1865(9):25042515.

74. Herrera Sanchez MB, Bruno S, Grange C, et al. Human liver stem cells and derived extracellular vesicles improve recovery in a murine model of acute kidney injury. Stem cell research \&3 therapy.2014;5(6):124.

75. Kholia S, Herrera Sanchez MB, Cedrino M, et al. Human liver stem cell-derived extracellular vesicles prevent aristolochic acid-induced kidney fibrosis. Frontiers in Immunology. 2018;9(JUL).

76. Zampetaki A, Kirton JP, Xu Q. Vascular repair by endothelial progenitor cells. Cardiovascular research. 2008;78(3):413-421.

77. Cantaluppi V, Gatti S, Medica D, et al. Microvesicles derived from endothelial progenitor cells protect the kidney from ischemia-reperfusion injury by microRNA-dependent reprogramming of resident renal cells. Kidney International. 2012;82(4):412-427.

78. Cantaluppi V, Medica D, Mannari C, et al. Endothelial progenitor cell-derived extracellular vesicles protect from complement-mediated mesangial injury in experimental anti-Thy1.1 glomerulonephritis.Nephrology Dialysis Transplantation. 2015;30(3):410-422.

79. Yang J, Wang M, Zhu F, et al. Putative endothelial progenitor cells do not promote vascular repair but attenuate pericyte-myofibroblast transition in UUO-induced renal fibrosis. Stem Cell Research and Therapy. $2019 ; 10(1)$.

80. Zhang H, Tao Y, Ren S, et al. Isolation and characterization of human umbilical cord-derived endothelial colony-forming cells. Exp Ther Med. 2017;14(5):4160-4166.

81. Burger D, Viñas JL, Akbari S, et al. Human Endothelial Colony-Forming Cells Protect against Acute Kidney Injury Role of Exosomes. American Journal of Pathology. 2015;185(8):2309-2323.

82. Viñas JL, Burger D, Zimpelmann J, et al. Transfer of microRNA-486-5p from human endothelial colony forming cell-derived exosomes reduces ischemic kidney injury. Kidney International.2016;90(6):1238-1250.

83. Vinas JL, Spence M, Gutsol A, et al. Receptor-Ligand Interaction Mediates Targeting of Endothelial Colony Forming Cell-derived Exosomes to the Kidney after Ischemic Injury. Sci Rep. 2018;8(1):16320.

84. Zhou Y, Xiong M, Niu J, et al. Secreted fibroblast-derived miR-34a induces tubular cell apoptosis in fibrotic kidney. Journal of Cell Science. 2014;127(20):4494-4506.

85. Li H, Xu Y, Zhang Q, Xu H, Xu Y, Ling K. Microvesicles containing mir-34a induce apoptosis of proximal tubular epithelial cells and participate in renal interstitial fibrosis. Experimental and Therapeutic Medicine. $2019 ; 17(3)$.

86. Zhang Y, Ma KL, Gong YX, et al. Platelet microparticles mediate glomerular endothelial injury in early diabetic nephropathy. Journal of the American Society of Nephrology.2018;29(11):2671-2695.

87. Wu XQ, Tian XY, Wang ZW, Wu X, Wang JP, Yan TZ. miR-191 secreted by platelet-derived microvesicles induced apoptosis of renal tubular epithelial cells and participated in renal ischemia-reperfusion injury via inhibiting CBS. Cell Cycle. 2019;18(2):119-129.

88. Pan T, Jia P, Chen N, et al. Delayed remote ischemic preconditioning confers renoprotection against septic acute kidney injury via exosomal miR-21. Theranostics. 2019;9(2):405-423.

89. Chen HH, Lai PF, Lan YF, et al. Exosomal ATF3 RNA attenuates pro-inflammatory gene MCP-1 transcription in renal ischemia-reperfusion.Journal of Cellular Physiology. 2014;229(9):1202-1211.

90. Jiang ZZ, Liu YM, Niu X, et al. Exosomes secreted by human urine-derived stem cells could prevent kidney complications from type i diabetes in rats. Stem Cell Research and Therapy. 2016;7(1). 
91. Jin J, Shi Y, Gong J, et al. Exosome secreted from adipose-derived stem cells attenuates diabetic nephropathy by promoting autophagy flux and inhibiting apoptosis in podocyte. Stem Cell Research and Therapy. 2019;10(1).

92. Zou X, Kwon SH, Jiang K, et al. Renal scattered tubular-like cells confer protective effects in the stenotic murine kidney mediated by release of extracellular vesicles. Scientific reports.2018;8(1):1263.

93. Wang H, Wang B, Zhang A, et al. Exosome-Mediated miR-29 Transfer Reduces Muscle Atrophy and Kidney Fibrosis in Mice. Molecular Therapy. 2019;27(3):571-583.

94. Fernandez-Martinez AB, Torija AV, Carracedo J, Ramirez R, de Lucio-Cazana FJ. Microparticles released by vascular endothelial cells increase hypoxia inducible factor expression in human proximal tubular HK-2 cells. The international journal of biochemistry \&6 cell biology. 2014;53:334-342.

95. Ranghino A, Bruno S, Bussolati B, et al. The effects of glomerular and tubular renal progenitors and derived extracellular vesicles on recovery from acute kidney injury. Stem Cell Research and Therapy. 2017;8(1):115.

96. Munkonda MN, Akbari S, Landry C, et al. Podocyte-derived microparticles promote proximal tubule fibrotic signaling via p38 MAPK and CD36. J Extracell Vesicles. 2018;7(1):1432206.

97. Zhao A, Kong F, Liu CJ, et al. Tumor cell-derived microvesicles induced not epithelial-mesenchymal transition but apoptosis in human proximal tubular (HK-2) cells: Implications for renal impairment in multiple myeloma. International Journal of Molecular Sciences.2017;18(3).

98. Zhang L, Wu X, Luo C, et al. The 786-0 renal cancer cell-derived exosomes promote angiogenesis by downregulating the expression of hepatocyte cell adhesion molecule. Molecular Medicine Reports.2013;8(1):272276 .

99. Chen G, Zhang Y, Wu X. 786-0 renal cancer cell line-derived exosomes promote 786-0 cell migration and invasion in vitro. Oncology Letters. 2014;7(5):1576-1580.

100. Jiang XL, Zhang Y, Tan B, Luo CL, Wu XH. Renal tumor-derived exosomes inhibit hepaCAM expression of renal carcinoma cells in a p-AKT-dependent manner. Neoplasma. 2014;61(4):416-423.

101. Wang L, Yang G, Zhao D, et al. CD103-positive CSC exosome promotes EMT of clear cell renal cell carcinoma: Role of remote MiR-19b-3p.Molecular Cancer. 2019;18(1).

Figure legends:

Figure 1: Study selection flow chart according to the PRISMA guidelines.

\section{Hosted file}

Table 1.docx available at https://authorea.com/users/353486/articles/477345-renoprotectiveeffects-of-extracellular-vesicles-a-systematic-review

\section{Hosted file}

Table 2.docx available at https://authorea.com/users/353486/articles/477345-renoprotectiveeffects-of-extracellular-vesicles-a-systematic-review

\section{Hosted file}

Table 3.docx available at https://authorea.com/users/353486/articles/477345-renoprotectiveeffects-of-extracellular-vesicles-a-systematic-review

\section{Hosted file}

Table 4.docx available at https://authorea.com/users/353486/articles/477345-renoprotectiveeffects-of-extracellular-vesicles-a-systematic-review 


\section{Hosted file}

Table 5.docx available at https://authorea.com/users/353486/articles/477345-renoprotectiveeffects-of-extracellular-vesicles-a-systematic-review

\section{Hosted file}

Table 6.docx available at https://authorea.com/users/353486/articles/477345-renoprotectiveeffects-of-extracellular-vesicles-a-systematic-review

\section{Hosted file}

Table 7.docx available at https://authorea.com/users/353486/articles/477345-renoprotectiveeffects-of-extracellular-vesicles-a-systematic-review

\section{Hosted file}

Table 8.docx available at https://authorea.com/users/353486/articles/477345-renoprotectiveeffects-of-extracellular-vesicles-a-systematic-review

\section{Hosted file}

Table 9.docx available at https://authorea.com/users/353486/articles/477345-renoprotectiveeffects-of-extracellular-vesicles-a-systematic-review

\section{Hosted file}

Table 10.docx available at https://authorea.com/users/353486/articles/477345-renoprotectiveeffects-of-extracellular-vesicles-a-systematic-review 
PRISMA 2009 Flow Diagram
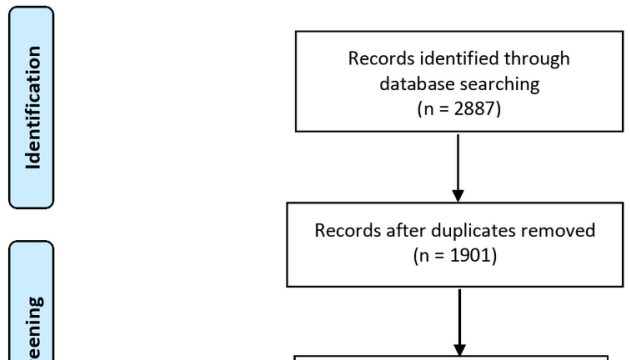

Records screened on basis of title and abstract $(n=1901)$

Records excluded ( $n=1773)$

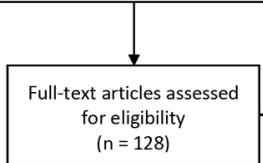

Full-text articles excluded, with reasons (n

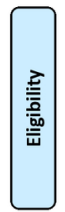
$=48$ )

Not in English languages (

$n=1)$

Not full text $(n=5)$

Conference abstract $(n=42)$

Studies included in qualitative synthesis $(n=80)$ 